\title{
El software empresarial como tecnología para el aprendizaje y el conocimiento: un enfoque experiencial
}

\author{
Eloy González-Acosta ${ }^{(1) \star}$, Maylié Almeida-González ${ }^{(2)}$ y Giceya C. Maqueira-Caraballo(3) \\ (1) Universidad de Guayaquil, Facultad de Ciencias Administrativas, Carrera de Contaduría Pública Autorizada, \\ Ciudadela Universitaria Ave. Delta SN y Avenida Kennedy, Guayaquil, Ecuador. (correo-e: eloygez@gmail.com) \\ (2) Universidad de Guayaquil, Facultad de Ciencias Administrativas, Carrera de Contaduría Pública Autorizada, \\ Ciudadela Universitaria Ave. Delta SN y Avenida Kennedy, Guayaquil, Ecuador. (correo-e: maylie.alm@gmail.com) \\ (3) Universidad de Guayaquil, Facultad de Educación Física y Recreación (FEDER), Ciudadela Universitaria, \\ Ave. Delta SN y Avenida Kennedy, Guayaquil, Ecuador. (correo-e: giceyamc@gmail.com) \\ * Autor a quien debe ser dirigida la correspondencia
}

Recibido Oct. 11, 2019; Aceptado Dic. 6, 2019; Versión final Dic. 24, 2019, Publicado Jun. 2020

\begin{abstract}
Resumen
La presente investigación tiene como objetivo establecer el impacto de un método de aprendizaje experiencial en los resultados académicos en estudiantes de contabilidad y auditoría. Este método se ha concebido impartiendo contenidos teóricos y prácticos apoyados en software empresariales. Se realizó un cuasiexperimento contrastado con la aplicación de una prueba estadística ANCOVA para determinar las diferencias en las calificaciones académicas en dependencia del método de aprendizaje y el horario de clases. Se realizó un cuestionario tipo Likert para evaluar actitud y la motivación. Los resultados de la prueba mostraron diferencias significativas en relación con el método de aprendizaje utilizado. Este trabajo contribuye a la literatura empírica sobre las tecnologías para la enseñanza y el aprendizaje experiencial, con la inserción del software empresarial como herramienta educativa con un marcado enfoque práctico.
\end{abstract}

Palabras clave: docencia universitaria; software empresarial; tecnologías; aprendizaje; conocimiento; experiencial

\section{Business software as technology for learning and knowledge: an experiential approach}

\begin{abstract}
This study tests the impact of an experiential learning method on the academic performance of accounting and auditing students. Imparting theoretical and practical content using business software developed the method. A quasi-experiment and an ANCOVA statistical test were used to determine the differences in academic performance depending on the learning method and class schedule. A Likert-type questionnaire was applied to evaluate attitude and motivation. The results showed significant differences in relation to the learning method used. This work contributes to the empirical literature on technologies for teaching and experiential learning by showing the practical application of business software as an educational tool.
\end{abstract}




\section{INTRODUCCIÓN}

Las Ilamadas Tecnologías de la Información y las Telecomunicaciones (TIC) han transformado la manera en que se ejecuta cualquier tipo de actividad humana. Según Berrío y Rojas (2014) los cambios tecnológicos radicales en los intercambios de información, configurando la economía de redes, información y conocimiento; han generado una sociedad global con capacidad masiva de intercambio de información a bajos costos, y procesos acelerados de innovación. La educación como ciencia ha sido una de las áreas del conocimiento que más impacto ha tenido con los acelerados avances tecnológicos ocurridos en los últimos años. Las TIC han revolucionado la forma en que se genera el proceso de aprendizaje de un estudiante. Son las tendencias modernas en el aprendizaje y la enseñanza más orientadas hacia el uso de una amplia gama de soporte tecnológicos que determinan que estos procesos sean más convenientes y atractivos tanto para los estudiantes como para los profesores. (Ivanović et al., 2018; Ausín et al., 2016). De esta forma se marca una evolución en la forma en que participa el docente y cómo gestiona el proceso educativo.

Hace una década la preparación de un estudiante universitario exigía el manejo de unas fuentes básicas mientras en la actualidad es imprescindible el manejo de múltiples fuentes de información. (Fernández et al., 2003). Para Domingo y Graells (2011) las TIC son capaces de ampliar la oferta informativa que multiplica las posibilidades para la orientación y tutorización, sobrepasando las limitaciones espaciales y temporales, de esta manera facilita el trabajo colaborativo y el autoaprendizaje, y potencia la interactividad y flexibilidad en el aprendizaje. El objetivo actual de las TIC es lograr aprendizajes superiores que generen el interés de los estudiantes donde el papel del docente sea solo el de orientador y moderador, más el papel protagónico recae en el propio estudiante (Sánchez et al., 2019)

Algunos estudios han separado las tecnologías utilizadas para la docencia enmarcándolas como Tecnologías del aprendizaje y el conocimiento (TAC). De esta manera se establece un campo que aborda los principios pedagógicos que demandan el uso de la tecnología y cómo poder investigar desde dentro la interacción entre enseñanza, aprendizaje y tecnología (Trujillo y Salvadores, 2019). Esta investigación está enmarcada dentro de los procesos de aprendizaje del estudiante universitario de Contabilidad y Auditoría, específicamente en la Facultad de Ciencias Administrativas de la Universidad de Guayaquil. En específico, se orienta a medir el impacto de tecnologías utilizadas en el campo profesional del contador, hacia el proceso formativo de estudiantes que cursan los últimos niveles de la carrera y que estarían próximos a insertarse en el mercado laboral.

Para Aguiar et al. (2018) en el uso de las TAC aún prevalece una función de mera sustitución de tecnologías, son versiones digitales de los tradicionales libros de texto y pizarras. En nuestro análisis bibliográfico hemos encontrado que no existe un estudio del uso del software empresarial en la enseñanza que lo identifique como una herramienta para el aprendizaje, limitando las TAC a la aplicación de las tecnologías a métodos convencionales de la educación. Por esta razón en este trabajo se pretende mostrar que determinadas herramientas tecnológicas no concebidas para la educación pueden considerarse como TAC, en específico para la formación del estudiante de Contabilidad y Auditoría.

El ejercicio profesional del contador está muy influenciado por la utilización de técnicas automatizadas que aumentan considerablemente la eficacia y la eficiencia de su trabajo y por ende de los resultados empresariales. Para Slobodan y Todorović (2015) el uso de tecnologías en el proceso contable-financiero puede reducir el tiempo necesario para las tareas tradicionales de registro a su vez que aumenta el tiempo disponible para el análisis de datos, el estudio del rendimiento y la elaboración de los informes estratégicos. Por todo esto, acercar estás herramientas al estudiante de contabilidad es mejorar su formación y sus competencias de cara al mercado laboral.

\section{OTROS ANTECEDENTES}

La inclusión de las TAC dentro del proceso educativo juega un papel en el aprendizaje como componente práctico desde un enfoque de aprendizaje experimental. Por esto es necesario recopilar algunos antecedentes sobre las TACs orientadas al proceso educativo y sobre el software empresarial como TAC.

\section{Las TACs orientadas al proceso educativo}

Para el proceso formativo moderno el docente tiene a su favor el uso de un grupo de herramientas tecnológicas que van desde los softwares de aplicación, dentro de los cuales se tienen los paquetes de ofimática; correo electrónico, procesamiento de textos, LMS, blogs, foros en línea, audio y podcasts de vídeo, wikis, plataformas de intercambio multimedia, voz sobre sistemas IP, juegos / simulaciones, portafolio electrónico y las redes sociales. (Seixas et al., 2014). Estás herramientas facilitan la creación de aulas virtuales donde es posible establecer una educación a distancia en la que, tanto docentes como estudiantes, no tienen que cumplir con requerimientos de presencia física. Y por lo cual, el alumno activo lidera su proceso de auto- aprendizaje, esfuerzo en el que se ve asistido por tutores y por sus propios compañeros de clase. (De Arco et al., 2017; Mendoza et al., 2019; Salazar et al., 2016). A partir de las condiciones del estudiante 
actual se considera que el uso generalizado de las computadoras, los Tablet, teléfonos y otros dispositivos móviles junto con la Web 2.0 ha abierto vías para la aplicación de herramientas de e-learning que se espera que cambie tanto la enseñanza y el aprendizaje. (Seixas et al., 2014; Soler et al., 2012).

Por otro lado, según De Arco et al. (2017) el b-learning consiste en adicionar a esta modalidad virtual a distancia sesiones presenciales, en una alternativa que pretende alcanzar un desarrollo más competitivo que pueda solventar las limitantes de la oferta académica por medio de estrategias y procesos de aprendizaje dinámico hacia una formación que más integral. Desde esta perspectiva diversos estudios han comprobado que el b-learning puede ser más efectivo que el aprendizaje presencial para formación por competencias (Nuñez y Monclúz, 2019). Este aprendizaje en línea permite alternativas más flexibles al modelo tradicional, que permiten al estudiante aprender sin restricciones de espacio o tiempo, mediante el uso de las diversas plataformas (Calderón y Ruiz, 2016).

Según García et al. (2018) el b-learning genera un campo de investigación que permitirá hallar herramientas y perspectivas nuevas que potencien la enseñanza y reduzcan los problemas del aprendizaje en una sociedad mediatizada como la que vivimos. Bajo este contexto se genera un ambiente personalizado de aprendizaje novedoso que se ha conceptualizado en los Personal Learning Enviroment (PLE). PLE es un nuevo concepto en el que los alumnos pueden gestionar su propio proceso de aprendizaje, colaborar y comunicarse con los demás y recibir el aprendizaje personalizado, por ejemplo, con el uso de las e-learning. (Abdellah, 2018).

Según Kompen et al. (2018) mediante el uso de un PLE, los estudiantes podrían controlar su proceso de aprendizaje con la capacidad de escoger y mezclar varias alternativas que les permiten capturar, clasificar, almacenar, analizar, generar, compartir, difundir y procesar la información, creando así el conocimiento. Se traduce como la respuesta a los enfoques pedagógicos, que exige que los propios alumnos deben tener el control sobre sus propios sistemas de e-learning. (Abdellah, 2018). Por lo tanto, hay un impacto en la transformación de la educación con utilización de nuevas metodologías y enfoques, pero también pueden ser útiles en la preparación de los alumnos de cara al mercado laboral (Kompen, 2018). Con la formación de un favorable PLE, el individuo, en su entorno personal, tiene la capacidad de autorregular su propio aprendizaje. (Chavez et al., 2015)

\section{El software empresarial como TAC}

Si bien hemos determinado que las TAC modifican el intercambio entre docentes y estudiantes en el proceso educativo, su concepción actual se establece como la transformación de los métodos tradicionales de enseñanza. Sin embargo, no suelen considerarse dentro de estas herramientas otras tecnologías no desarrolladas con finen educativos o para la difusión de la información y que pueden ser también recursos claves en el aprendizaje del estudiante. Para el caso del estudiante de Contabilidad y Auditoría un elemento tecnológico que puede incluirse para el aprendizaje del estudiante es el software empresarial, teniendo en cuenta su extendida utilización en cualquier organización para su control, planeación y organización de todos sus procesos. Según Nori et al. (2016) el uso de un software contable puede proporcionar a los educadores que lo emplean como instrumento pedagógico un enfoque más eficaz para facilitar la comprensión de los estudiantes de las bases teóricas y las partes técnicas de contabilidad.

El acercamiento del estudiante a estos sistemas representa la integración de los conceptos y principios de la profesión con las herramientas que en la práctica se utilizan en el trabajo profesional. Cuando el docente es capaz de insertar el uso de estas tecnologías incide de manera favorable en la comprensión de los conceptos que se adquieren desde un enfoque práctico y genera habilidades que serán ya competencias del futuro profesional. Se propicia el aprendizaje dentro de un entorno completamente experiencial. Tanto los softwares contables como de auditoría influyen en la mejor comprensión de contenido. En el trabajo de Becker et al. (2016) se determina el impacto del software IDEA en el aprendizaje de estudiantes, teniendo en cuenta que van a obtener el tipo de conocimiento profundo y duradero necesario, que podrá usar años después de que termine su curso de auditoría.

Hill (2015) recomienda la enseñanza de softwares contables para la capturar y el procesamiento de datos de transacciones comerciales comunes y la preparación informes básicos estandarizados, que proporciona al estudiante además de habilidades informáticas, la comprensión de la organización y de los flujos de los datos registrados. Según Kolb (2014), quien da las primeras definiciones del aprendizaje experiencial, las personas aprenden sobre todo de su experiencia y los resultados de ese aprendizaje pueden ser evaluadas de manera muy confiable. Los programas del aprendizaje experiencial se utilizan de forma extendida en la educación superior con la inclusión de pasantías, prácticas de campo, tareas de trabajo-estudio, juegos de rol, simulaciones de negocios y otras formas de educación basada en la experiencia que desempeñan un importante papel en la formación de los estudiantes. El aprendizaje experiencial es la manifestación práctica de la enseñanza donde los estudiantes participan activamente en el proceso y los resultados del aprendizaje son estimulados por la experiencia (Wright et al., 2018; Rosales et al., 2016). 
Para el caso de los estudiantes de contabilidad y auditoría el aprendizaje con los softwares empresariales implica situarlos en un EPL donde se simulan operaciones reales. Ali' et al. (2019) presentó resultados significativos recreando el ambiente de oficina para el estudiante de Contabilidad, donde simularon las condiciones laborales de marcaje de reloj y permanencia en espacios con computadoras, sillas y archivos similares a la oficina con el acompañamiento de un docente. Finalmente concluye que un método de aprendizaje experiencial que expone a los estudiantes a las imágenes reales del mundo de la contabilidad para mejorar las habilidades técnicas y no técnicas de los estudiantes (Ali' et al., 2019). En importante que el estudiante de contabilidad se coloque en una situación donde intentaría resolver problemas de negocios del tal cual se presenta en mundo empresarial real, manejando las situaciones de negocios y la terminología que se utiliza en la práctica. (Abbott y Palatnik, 2017). Boulianne (2014) presentó la investigación donde comparó los resultados de la adquisición de conocimiento de estudiantes de contabilidad mediante un caso de contabilidad manual contra otro donde utilizaron un software. En sus resultados sugiere que la mejor manera para que los estudiantes adquieran conocimientos concretos del ciclo contable está completando casos utilizando métodos manuales e informáticos.

Consideramos que a pesar de los múltiples estudios que vinculan las tecnologías con la enseñanza, las investigaciones se orientan en general al impacto tecnológico en la relación docente-estudiante y a las nuevas condiciones en que se realiza el aprendizaje. No se ha profundizado en el uso de tecnologías provenientes del ejercicio profesional, y en los estudios realizados no se ha planteado establecerlo como un método de aprendizaje. Nuestro trabajo pretende, como novedad, colocar a soluciones tecnológicas empresariales como herramientas formalmente didácticas, que deben ser consideradas dentro de la revolución que representan las nuevas tecnologías en la educación, conceptualizadas dentro del aprendizaje experiencial. Bajo la premisa que no es lo mismo aprendizaje experiencial que formación práctica (Kolb, 2014).

En el presente trabajo pretendemos corroborar que la utilización del software empresarial en las aulas con el estudiante de contabilidad y auditoría en la Universidad de Guayaquil tiene un impacto en la adquisición de sus conocimientos y genera un acercamiento a situaciones laborales reales. De esta manera definir este tipo de software como una TAC, siempre y cuando se utilice en este EPL. A la vez determinarlo como metodología dentro del campo de estudio del aprendizaje experiencial. Se utilizan dos softwares empresariales en versiones de prueba o demostrativa: el software de contabilidad Odoo y el software de auditoría IDEA. Se utilizaron como aprendizaje alternativo al método tradicional de impartir los contenidos mediante clases magistrales y prácticas sin el uso de estas tecnologías.

Odoo es uno de los Enterprise Resource Planning (ERP) más conocidos y utilizados. Los ERP son programas para gestionar mediante varios módulos los diferentes procesos de una empresa para alcanzar una administración integrada (Adhi et al., 2019). En nuestro caso utilizaremos en el aula una versión gratuita de un módulo contable de Odoo orientado al registro contable de operaciones en pequeñas y medianas empresas y generar informes financieros. Con el procesamiento en Odoo se realizarán operaciones simuladas vinculadas con los objetivos de la materia de contabilidad. IDEA, por su parte, es un software diseñado para la importación de datos desde sistemas de información financiera y su análisis para apoyar el trabajo de un auditor, mediante la búsqueda de errores y fraudes con la identificación de relaciones, patrones, valores atípicos y anomalías en sus datos (Beaker, 2016). Se utiliza una versión gratuita de IDEA para evaluar en el aula datos simulados y vincularlos con los conceptos de la materia auditoría. Nuestra investigación consiste en presentar contenidos de contabilidad y de auditoría apoyados en casos prácticos con Odoo e IDEA respectivamente. De manera que se genere un ambiente donde el estudiante se coloque en situaciones de trabajo de campo y de oficina propias del profesional contable y de auditoría.

Se establece como objetivo determinar si existen diferencias entre los resultados académicos de estudiantes dependiendo del método de aprendizaje, sea el método experiencial o el método tradicional. Bajo esta premisa, buscar una posible relación con el horario de clase de los estudiantes, (nocturnos o diurnos), teniendo en cuenta que el estudiante nocturno tiene una relación laboral y por tanto puede contar con más referencias prácticas de los contenidos impartidos. Para realizar un análisis preciso determinar la afectación de factores como la motivación y actitud de los estudiantes; así como los resultados académicos del semestre anterior, considerando que pueden arrastrarse falencias de conocimiento. El objetivo de la investigación, en este caso, es conocer si existen diferencias en el resultado académico de los estudiantes de contabilidad y auditoría aplicando dos métodos de aprendizaje, el método tradicional o un método experiencial; en interacción con el horario de clases del estudiante. A su vez controlar el resultado académico del período anterior.

\section{METODOLOGÍA}

Se desarrolla un diseño cuasiexperimental para demostrar la relación entre las diferentes variables para los ciclos educativos entre el 2018 y 2019. Al diseñar un cuasiexperimento se busca la manipulación de variables sin aspirar el nivel de rigurosidad de un experimento puro, nuestra limitación es la imposibilidad de formar grupos aleatorios que obliga a trabajar con los estudiantes en sus grupos intactos de clases (Hernández et 
al., 2014). Hemos considerado que esto puede implicar un riesgo de contaminación de determinadas variables, que regulamos con la selección de un método estadístico apropiado (Huitema, 2011). Al estudiar las diferencias en las variables en un momento único asumimos una investigación transeccional correlacional, descartando para este caso un análisis longitudinal (Hernández et al., 2014).

La muestra se toma de los estudiantes de la carrera de Contaduría Pública Autorizada de la Facultad de Ciencias Administrativa de la Universidad de Guayaquil, dividida en ocho niveles o semestres. En el estudio participaron 483 estudiantes de los niveles sexto y octavo, que representan el $24 \%$ de los 2028 que cursan la carrera durante el semestre correspondiente al ciclo II del período 2018-2019. Los grupos corresponde a la clase y aula a los que está incluido cada estudiante por matriculación y que no se ha modificado en la investigación. La muestra se distribuye como se muestra en la Tabla 1.

El cuasiexperimento se realiza aplicando a los grupos experimentales un método de aprendizaje experiencial, que en este caso consiste en impartir los contenidos apoyados en el uso de software empresariales. Para el sexto nivel en los 4 grupos los temas se impartieron para la materia de Contabilidad especial y se desarrollan mediante el uso del software ERP contable Odoo, en una versión de prueba. Para el octavo nivel los 5 grupos experimentales recibieron los contenidos de la materia Dictámenes de Auditoría con el uso del software de auditoría IDEA, también en una versión de prueba.

Tabla 1: Distribución de la muestra y de los grupos.

\begin{tabular}{|c|c|c|c|c|c|c|c|c|}
\hline \multirow{2}{*}{ Tipo de Grupo } & \multirow{2}{*}{$\begin{array}{l}\text { Método } \\
\text { Aplicado }\end{array}$} & \multirow{2}{*}{ Estudiantes } & \multirow{2}{*}{$\begin{array}{c}\text { Grupos 6to } \\
\text { Nivel }\end{array}$} & \multirow{2}{*}{$\begin{array}{l}\text { Grupos } \\
\text { 8vo Nivel }\end{array}$} & \multicolumn{2}{|c|}{ Sexo } & \multicolumn{2}{|c|}{ Horario } \\
\hline & & & & & $F$ & $M$ & Diurno & Nocturno \\
\hline Grupo Control & Tradicional & 221 & 4 & 3 & 147 & 74 & 70 & 151 \\
\hline Grupo Experimental & Experiencial & 262 & 4 & 5 & 179 & 83 & 69 & 193 \\
\hline Total & & 483 & 8 & 8 & 326 & 157 & 139 & 344 \\
\hline
\end{tabular}

Las clases se presentan simulando situaciones donde el estudiante conecta con las teorías y elementos prácticos en un ambiente que recrea el escenario real dentro de una organización donde se realizan tareas relacionadas al trabajo profesional del contador-auditor, en los cuales el uso de programas informáticos aplicados es cada vez más común. En los grupos de control se utiliza un método de aprendizaje tradicional. Tanto para los 4 grupos de sexto nivel que recibieron la materia de Contabilidad Especial y los 3 grupos de octavo nivel que les corresponde la materia de Dictámenes de Auditoría, los contenidos se presentan separados en un componente docente de clases magistrales y un componente práctico que incluye el desarrollo de talleres, casos prácticos y otros trabajos colaborativos. Los grupos de control no recibieron contenidos vinculados con softwares relacionados a la especialidad. Para los estudiantes de los grupos control y experimentales se tomó el mismo examen de forma simultánea y este consistió en preguntas de opción múltiple sobre los contenidos que son objetivo del programa de cada materia para el semestre, para los estudiantes de sexto un examen de contabilidad y de auditoría para el octavo.

Se realiza un estudio de los resultados académicos de los estudiantes de ambos grupos, los que reciben los contenidos mediante el método de aprendizaje experiencial y por el método de aprendizaje tradicional. Estos resultados se obtienen a partir de la calificación promedio conseguidas en el examen parcial de la materia de Contabilidad Especial para el sexto nivel y Dictámenes de Auditoría para el octavo. Se analiza, además los resultados académicos de cada estudiante en el semestre anterior en la materia prerrequisito que para el sexto nivel es Contabilidad superior II y para el octavo nivel es Auditoría III, con el objetivo de observar los resultados anteriores de las calificaciones que pudieran mostrar diferencias en la solidez de conocimientos de los estudiantes previos a la aplicación de la investigación. Tanto las calificaciones actuales como las del semestre anterior corresponden a notas de examen en una escala de 0 a 10 puntos.

Se ha procedido a realizar una prueba estadística de Análisis de covarianza (ANCOVA), que permita determinar diferencias de medias en las variables descritas. Específicamente las diferencias significativas en las calificaciones obtenidas por los estudiantes (Variable Dependiente) en dependencia del método de aprendizaje utilizado (Variable Dependiente), con interacción del horario de clases del estudiante (Variable Dependiente) y controlando la calificación del semestre anterior (Covariable). Adicionalmente a este resultado se realiza una valoración de la potencia estadística. No se confirmarán los supuestos de las pruebas paramétricas porque, en cualquier caso, no existe una variante de prueba no paramétrica equivalente al ANCOVA.

Teniendo en cuenta que nuestro análisis no es un experimento puro, sobre todo porque no se existe una asignación aleatoria de los elementos a los grupos, consideramos la prueba ANCOVA y los modelos de regresión relacionados, los cuales son casi siempre apropiados para este diseño porque la covariable atrapa toda la información utilizada para asignar sujetos a los tratamientos. (Huitema, 2011). Teniendo en cuenta la 
naturaleza del cuasiexperimento, para reducir la posible incidencia de otras variables se estudian los factores actitud y motivación. La intención es establecer que tanto para los estudiantes de los grupos control como experimentales la actitud y la motivación ante el estudio son similares y no distorsionan significativamente la relación entre las calificaciones y el método de aprendizaje utilizado.

Para esto se elabora y aplica un cuestionario que adopta una escala de Likert, formado por 24 preguntas en una escala de 1 a 5 equivalente a Nunca, Casi nunca, A veces, Casi siempre y Siempre (Hernández et al., 2014). La suma de los resultados del cuestionaros se agrupa según cinco categorías por separado en Muy desfavorable, Desfavorable, Indiferente, Favorable y Muy favorable; para la evaluación de la actitud y la motivación. Para evaluar la fiabilidad de los resultados obtenidos en los cuestionarios aplicados se ha calculado el alfa de Cronbach. Las preguntas elaboradas aparecen la Tabla 2.

Se elabora el cuestionario con los ajustes propuestos por un grupo de seis docentes con amplia experiencia en investigación relacionadas a las ciencias de la educación, los cuales revisaron el contenido, orden y pertinencia de las preguntas. Tanto la motivación como la actitud son constructos hipotéticos, que no pueden ser observados directamente, pero que han revelado tener un importante poder explicativo y predictivo en las conductas humanas, y, por supuesto, en las adoptadas por los estudiantes (Delgado y Castrillo, 2015). Por esta razón se considera pudieran influir en las variables que se estudian y disminuir el rigor de los efectos. El análisis estadístico de los datos en general se realiza con el Software estadístico SPSS V23, utilizado para el estudio de los datos, alfa de Cronbach y escala de Likert y las diferencias determinadas en el ANCOVA. Para el cálculo de la potencia estadística se utiliza el software G*Power 3.1.9.2.

\section{RESULTADOS Y DISCUSIÓN}

El análisis de los cuestionarios aplicados, como se muestra en la Tabla 3, arrojó que el $95 \%$ en el grupo de control y el $93 \%$ en el experimental tienen una actitud ante el estudio entre favorable y muy favorable. Por otra parte, la motivación de los estudiantes resultó por encima de favorable en un $95 \%$ para el grupo control y de $96 \%$ para el experimental. Si bien los resultados no son idénticos para los grupos, los investigadores consideran que los valores porcentuales son muy similares y no tendrían efecto en las calificaciones de los estudiantes, lo que permite un escenario adecuado para medir el efecto de otras variables. El cálculo del Alfa de Cronbach fue de 0,860 que interpretamos de buena confiabilidad.

Tabla 2: Cuestionario escala de Likert para la evaluación de la motivación y la actitud hacia el aprendizaje.

\begin{tabular}{|c|c|c|c|}
\hline \multicolumn{2}{|r|}{ Actitud hacia el aprendizaje } & \multicolumn{2}{|r|}{ Motivación hacia el aprendizaje } \\
\hline Código & Pregunta & Código & Pregunta \\
\hline $\mathrm{A} 01$ & $\begin{array}{l}\text { Suelo implicarme en las actividades } \\
\text { académicas }\end{array}$ & M01 & $\begin{array}{l}\text { Decidí estudiar esta carrera para trabajar en algo } \\
\text { que me gusta }\end{array}$ \\
\hline A02 & $\begin{array}{l}\text { Considero importante obtener buenas } \\
\text { calificaciones }\end{array}$ & M02 & $\begin{array}{l}\text { Estudio para aumentar mis posibilidades en el } \\
\text { mercado de trabajo }\end{array}$ \\
\hline A03 & Me preocupa fracasar en mis estudios & M03 & $\begin{array}{l}\text { Estudio para aprender y avanzar en mis } \\
\text { conocimientos }\end{array}$ \\
\hline A04 & Estudiar no me genera ansiedad & M04 & $\begin{array}{l}\text { Me motiva comprobar que soy capaz de superarme } \\
\text { a mí mismo }\end{array}$ \\
\hline A05 & $\begin{array}{l}\text { No acostumbro a retrasarme en la } \\
\text { realización de las tareas académicas }\end{array}$ & M05 & Prefiero llevar una vida ordenada, organizada \\
\hline A06 & $\begin{array}{l}\text { Pregunto frecuentemente a mis profesores } \\
\text { sobre mis calificaciones }\end{array}$ & M06 & Estudio para educarme, para madurar como persona \\
\hline A07 & Me preocupa hacer todo bien & M07 & $\begin{array}{l}\text { Estudio porque así puedo vivir con más } \\
\text { independencia }\end{array}$ \\
\hline A08 & $\begin{array}{l}\text { Considero que todos los contenidos son } \\
\text { importantes }\end{array}$ & M08 & $\begin{array}{l}\text { Estudio por vocación, porque siempre quise estudiar } \\
\text { esta carrera }\end{array}$ \\
\hline A09 & $\begin{array}{l}\text { Priorizo mi tiempo en función de mis estudios } \\
\text { sobre otras actividades }\end{array}$ & M09 & $\begin{array}{l}\text { Estudio porque me hace sentirme bien conmigo } \\
\text { mismo/a }\end{array}$ \\
\hline A10 & $\begin{array}{l}\text { Dedico tiempo a todas las tareas sean fáciles } \\
\text { o complejas }\end{array}$ & M10 & $\begin{array}{l}\text { Estudio para conocer gente nueva y entablar } \\
\text { amistades con chicos como yo }\end{array}$ \\
\hline A11 & $\begin{array}{l}\text { Considero que estoy capacitado para asumir } \\
\text { tareas complejas }\end{array}$ & M11 & $\begin{array}{l}\text { Mis profesores y su metodología me motivan para } \\
\text { seguir estudiando }\end{array}$ \\
\hline A12 & $\begin{array}{l}\text { Siento que tengo una buena base adquirida } \\
\text { en niveles anteriores }\end{array}$ & M12 & $\begin{array}{l}\text { Estudio en la universidad porque me divierto también } \\
\text { mucho }\end{array}$ \\
\hline
\end{tabular}


Tabla 3: Resultados porcentuales de la aplicación del cuestionario escala Likert para Actitud y Motivación en los estudiantes de sexto y octavo separados en los grupos control y experimentales.

\begin{tabular}{|l|c|c|c|c|}
\hline \multirow{2}{*}{ Evaluación } & \multicolumn{2}{|c|}{ Actitud } & \multicolumn{2}{c|}{ Motivación } \\
\cline { 2 - 5 } & Grupo Control & Grupo Experimental & Grupo Control & Grupo Experimental \\
\hline Muy Desfavorable & $0 \%$ & $0 \%$ & $0 \%$ & $0 \%$ \\
\hline Desfavorable & $0 \%$ & $0 \%$ & $1 \%$ & $0 \%$ \\
\hline Indiferente & $5 \%$ & $7 \%$ & $4 \%$ & $3 \%$ \\
\hline Favorable & $15 \%$ & $16 \%$ & $8 \%$ & $12 \%$ \\
\hline Muy Favorable & $80 \%$ & $77 \%$ & $87 \%$ & $84 \%$ \\
\hline
\end{tabular}

La prueba estadística ANCOVA presentó un resultado para el modelo de $F(1.478)=4,723$, sig. $=0.030$ y la potencia estadística se determinó en $\beta=0.99, \mathrm{f}=0.33$. Como aparece en la Tabla 4 se obtuvo un resultado de $p<0.05$ lo que indica que efectivamente existen diferencias significativas entre los resultados académicos de los estudiantes y el método de aprendizaje que se utilice, en este caso un método de aprendizaje experiencial o el método tradicional, con interacción del horario de clase. La evaluación previa de motivación y actitud; así como el control de las notas anteriores como covariable en la prueba ANCOVA nos permite establecer que estos factores no contaminan los efectos en la variable dependiente para establecer una correlación. Consideramos que este análisis resuelve las limitaciones inherentes a un cuasiexperimento. Bajo estas condiciones podemos determinar una relación causal entre el método de aprendizaje y los resultados académicos.

Dentro del modelo la variable control de calificación obtenida en el semestre anterior muestra una sig $=.001$, que prueba su relación con la interacción de las variables. Se determina el coeficiente Eta parcial al cuadrado para calcular el tamaño del efecto de una variable cuantitativa en una categórica dentro de estudios de análisis de varianzas. Se considera un resultado de poco efecto para un Eta en torno a 0.01, medio sobre el 0.06 y alto sobre los 0.14 (Huitema, 2011). La tabla 4 muestra un Eta parcial al cuadrado con efecto alto en todas las relaciones estudiadas. Al determinar mediante el paquete estadístico $G^{*}$ Power un valor de $\beta$ al $99 \%$ entendemos que son mínimas las posibilidades de cometer el error tipo II y muy altas de aceptar la hipótesis alternativa siendo verdadera.

Tabla 4: Prueba de los efectos inter-sujetos resultado del ANCOVA, con Variable dependiente: Calificación actual. R al cuadrado $=0,334$ ( $\mathrm{R}$ al cuadrado ajustada $=0,329)$

\begin{tabular}{|l|c|c|c|c|c|c|}
\hline Origen & $\begin{array}{l}\text { Tipo III de suma de } \\
\text { cuadrados }\end{array}$ & GI & $\begin{array}{l}\text { Media } \\
\text { cuadrática }\end{array}$ & F & Sig. & $\begin{array}{l}\text { Eta parcial al } \\
\text { cuadrado }\end{array}$ \\
\hline Modelo corregido & 195,746 & 4 & 48,936 & 59,989 &, 000 &, 334 \\
\hline Intersección & 238,142 & 1 & 238,142 & 291,927 &, 000 &, 379 \\
\hline Calificación_anterior & 23,624 & 1 & 23,624 & 28,960 &, 000 &, 057 \\
\hline Método_Aprendizaje & 54,276 & 1 & 54,276 & 66,535 &, 000 &, 122 \\
\hline Horario & 50,160 & 1 & 50,160 & 61,489 &, 000 &, 114 \\
\hline Método_Aprendizaje * Horario & 3,853 & 1 & 3,853 & 4,723 &, 030 &, 010 \\
\hline Error & 389,933 & 478 &, 816 & & & \\
\hline Total & 31021,130 & 483 & & & & \\
\hline Total corregido & 585,679 & 482 & & & & \\
\hline
\end{tabular}

En una comparación de resultados, Boulianne (2014) encontró diferencias significativas al comparar el aprendizaje de los estudiantes de contabilidad que utilizaron un método de registro contable manual con otro que utilizó softwares contables, aunque su estudio era de tipo no experimental y su analisis se enmarcó exclusivamente en los resultados obtenidos en la aplicación de cuestionarios. En su caso el estudio comparaba otras variables como la edad y nacionalidad de los estudiantes. De igual forma Becker et al. (2016) determina un rendimiento mayor en estudiantes de contabilidad en materia de auditoría utilizando archivos de datos analizados mediante el software IDEA, en una investigación más orientada a medir las capacidades de los estudiantes en el manejo de las computadoras. Aunque reconoce la incidencia de este tipo de software en el desarrollo de las auditorías. Ali' et al., (2019) determinó un nivel de éxito mayor en estudiantes de contabilidad con un método de aprendizaje que llamaron Aprendizaje experiencial de contabilidad en firmas, donde se a los alumnos se sometieron a contenidos en un ambiente simulado de computadoras similar al de una empresa. Delgado y Castrillo (2013), en su estudio comparativos de métodos de aprendizaje evalúa factores de dedicación y motivación, además otros como género y edad. 
Adicionalmente realizamos el mismo análisis del ANCOVA, pero eliminando el efecto de la covariable Calificación del semestre anterior, esto es equivalente a una prueba ANOVA de doble clasificación. EI resultado de este modelo fue de $F(1,479)=1,650$. Esto nos indicó que al incluir la covariable en el modelo el valor de $\mathrm{F}$ fue superior lo que significa que se explicó mucho más la varianza al incorporar el efecto de la calificación anterior en el modelo. Delgado y Castrillo (2013) encontraron diferencias significativas al controlar covariables como dedicación y motivación determinando su incidencia en el modelo ANCOVA. En nuestra investigación los factores actitud y motivación, teniendo en cuenta la naturaleza de estas variables no continuas, se consideró no incluirlos como covariables a controlar dentro del modelo de análisis de covarianza y solamente evaluar que los resultados sean similares para los grupos y no representen una distorsión importante de los efectos.

El análisis de las medias marginales estimadas que aparece en la Tabla 5 muestra las diferencias de la calificación obtenida por los estudiantes tanto en relación con el horario de clases como el método de aprendizaje aplicado. Los estudiantes del horario diurno que recibieron los contenidos por el método experimental basado en la integración de los softwares empresariales terminaron con calificaciones de una media de 8,701 mientras la media de los que recibieron la materia por el método tradicional fue de 7,197. Para el horario nocturno la media de las puntuaciones para el grupo experimental fue 8,180 mientras con el método tradicional la media de los resultados fue de 7,197. Si bien la media de las calificaciones es superior en los estudiantes que recibieron los contenidos por el método de aprendizaje experiencial sobre los que recibieron por el método tradicional, estas diferencias son a su vez mayores para el horario diurno que nocturno.

Tabla 5. Análisis de las medias marginales estimadas por Método de aprendizaje y el horario de clases. Para Variable dependiente: Calificación actual. Las covariables que aparecen en el modelo se evalúan en los valores siguientes: Calificación Anterior = 8,1447.

\begin{tabular}{|l|l|c|c|c|c|}
\hline \multirow{2}{*}{ Método de Aprendizaje } & \multirow{2}{*}{ Horario } & \multirow{2}{*}{ Media } & \multirow{2}{*}{$\begin{array}{c}\text { Error } \\
\text { estándar }\end{array}$} & \multicolumn{2}{|c|}{ Intervalo de confianza al 95\% } \\
\cline { 5 - 6 } & & &, 113 & Límite inferior & Límite superior \\
\hline \multirow{2}{*}{ Aprendizaje Experiencial } & Diurno & 8,701 & 8,478 & 8,923 \\
\cline { 2 - 6 } & Nocturno & 8,180 &, 065 & 8,052 & 8,308 \\
\hline \multirow{2}{*}{ Aprendizaje Tradicional } & Diurno & 8,119 &, 109 & 7,905 & 8,333 \\
\cline { 2 - 6 } & Nocturno & 7,197 &, 075 & 7,051 & 7,344 \\
\hline
\end{tabular}

Nuestros resultados, que muestran un rendimiento mayor en estudiantes con los que se utiliza un aprendizaje apoyado en software empresariales en comparación a métodos tradicionales, coinciden con los de Boulianne (2014) y Becker et al. (2016). Consideramos además que nuestros resultados coinciden con los de Ali' et al., (2019), teniendo en cuenta que nuestro método de aprendizaje experiencial simula una situación de oficina que genera mejores resultados académicos, similares a los obtenidos por estos autores. Delgado y Castrillo (2013) realizan una investigación con una metodología similar pero el estudio se enfoca a evaluar un método de aprendizaje cooperativo.

\section{CONCLUSIONES}

Los resultados empíricos derivados de la aplicación de las técnicas aplicadas sobre las calificaciones de los estudiantes de las materias de Contabilidad Especial, para los alumnos de sexto nivel, y de Dictámenes de Auditoría para estudiantes de octavo, en dependencia de utilizar un método de aprendizaje experiencial o un método de aprendizaje tradicional permiten arribar a las conclusiones siguientes: 1) Existen diferencias significativas entre los resultados académicos y el método de aprendizaje utilizado, al controlar las calificaciones obtenidas en el semestre anterior aparece que efectivamente tiene influencia en los resultados del modelo; 2) Las medias de las calificaciones obtenidas aplicando un aprendizaje experiencial mostraron resultados superiores en comparación con el tradicional y los estudiantes de horario diurno obtuvieron notas más altas con respecto a los que reciben sus clases en horario nocturno; 3) Del análisis de los cuestionarios aplicados se encontró que para ambos grupos de estudiantes la actitud y la motivación son favorables y el escenario para contrastar del cuasiexperimento eran adecuadas; 4) Al establecer una influencia en la aplicación del software en los resultados se convierte en una técnica para la enseñanza y, por su naturaleza práctica como herramienta propia del mundo empresarial, donde el estudiante aprende en un ambiente que simula el ejercicio profesional, lo definimos como una variante para el aprendizaje experiencial en los estudiantes de contabilidad y auditoría; 5) Se presenta a docentes e investigadores el uso de herramientas profesionales que seguramente muchos han utilizado para impartir contenidos académicos, pero en este caso, abordadas desde una perspectiva formalmente pedagógica, con una intención de definirla desde la didáctica y el aprendizaje. 


\section{REFERENCIAS}

Abbott, J. I., y Palatnik, B.R., Students' perceptions of their first accounting class: implications for instructors, https://doi.org/10.1080/09639284.2017.1381032, Accounting Education, 27(1), 72-93 (2017)

Abdellab, I.M., The effect of personal learning environments on participants' higher order thinking skills and satisfaction, https://doi.org/10.1080/14703297.2018.1534601, Innovations in Education and Teaching International, 56(4), 1-16 (2018)

Ali', R., Baharudin, I., y Nori, W., Accounting Experiential Learning Firm (AELF): Learning Accounting in an Office Setting, https://doi.org/10.18421/TEM82-50, TEM Journal, 8(2), 686-691 (2019)

Adhi, M., Purwanto, R., Sugiarto, A., Heru, A., Rokhmawan, T., Designing odoo's enterprise resource planning in Micro, Small and Medium Enterprises (MSMEs), https://doi.org/10.1088/1742-6596/1175/1/012202, Journal of Physics: Conference Series, 1175(1), 1-8 (2019)

Aguiar, B.O., Velázquez, R.M., y Aguiar, J.L., Innovación docente y empleo de las TIC en la Educación Superior, Revista Espacios, 40(2), 8-20 (2019)

Ausín, V., Abella, V., Delgado, V., y Hortigüela, D., Aprendizaje basado en proyectos a través de las TIC. Una experiencia de innovación docente desde las aulas universitarias, https://doi.org/10.4067/S0718-50062016000300005, Formación Universitaria, 9(3), 31-38 (2016)

Becker, D., Pernsteiner, A., y Drum, D., The roles of technology acceptance and computer learner aptitude on accounting student learning achievement with IDEA software, https://doi.org/11.270.10.1504/IJLT.2016.081709, International Journal of Learning Technology, 11(4), 270-284 (2016)

Berrío, C., y Rojas, H., La brecha digital universitaria: La apropiación de las TIC en estudiantes de educación superior en Bogotá (Colombia), Comunicar (en línea), XXI (43), 133-142 (2014).

Boulianne, E., Impact of accounting software utilization on students' knowledge acquisition, https://doi.org/10.1108/JAOC12-2011-0064, Journal of Accounting \& Organizational Change, 10(1), 22-48 (2014)

Calderón, C., Ruiz, J., y Sánchez, J., Integración de las tecnologías de la información y comunicación en un modelo de enseñanza flexible: El caso del centro universitario de los valles de Guadalajara, México, https://doi.org/10.4067/S071850062016000500005, Formación universitaria, 9(5), 37-48 (2016)

Chaves, E., Trujillo, J.M., y López, J.A, Autorregulación del aprendizaje en entornos personales de aprendizaje en el grado de educación primaria de la Universidad de Granada, España, https://doi.org/10.4067/S0718-50062015000400008, Formación universitaria, 8(4), 63-76 (2015)

De Arco, L.K., Barrio, H., y Parra, G., Análisis de factibilidad de un sistema de educación b-learning: caso de estudio Universidad de San Buenaventura, https://doi.org/10.18359/ravi.2706, Revista Academia y Virtualidad, 10(2), 7-19 (2017)

Delgado, M., y Castrillo, L.A., Efectividad del aprendizaje cooperativo en contabilidad: una contrastación empírica, Revista de Contabilidad (en línea), 18(2), 138-147 (2015).

Domingo, M., y Graells, P., Aulas 2.0 y uso de las TIC en la práctica docente, https://doi.org/10.3916/C37-2011-03-09, Comunicar, XIX(37), 169-175 (2011)

Fernández, M.C., Cebreiro, B., y Fernández, J.C., Competencias para el aprendizaje en red de los alumnos de educación secundaria en Galicia, Pixel-Bit. Revista de Medios y Educación [en linea], 38, 7-21 (2011).

Garcia, R., Aguaded, I., y Bartolomé, A., La revolución del blended learning en la educación a distancia, https://doi.org/10.5944/ried.21.1.19803, RIED Revista Iberoamericana de Educación a Distancia, 21(1), 25-32 (2018)

Hernández, R., Fernández, C., y Baptista, P., Metodología de la investigación, 6ta ed., McGraw-Hill, México D.F, México (2014)

Hill, M.C., Teaching transaction processing using trial-version software in accounting information systems courses, https://doi.org/10.1016/S1085-4622(07)08001-7, Advances in Accounting Education Teaching and Curriculum Innovations, 8, 1-23 (2015)

Huitema, B., The Analysis of Covariance and Alternatives: Statistical Methods for Experiments, Quasi-Experiments, and Single-Case Studies, 2nd Edition, Wiley, New Jersey, USA (2011)

Ivanović, M., Milićević, A.K., y otros tres autores, Experiences and perspectives of technology-enhanced learning and teaching in higher education-Serbian case, https://doi.org/10.1016/j.procs.2018.08.086, Procedia Computer Science, 126, 1351-1359 (2018)

Kolb, D.A., Experiential learning: Experience as the source of learning and development, FT press, NJ, USA (2014)

Kompen, R.T., Edirisingha, P., y otros tres autores, Personal learning environments based on web 2.0 services in higher education, https://doi.org/10.1016/j.tele.2018.10.003, Telematics and Informatics, 38(5), 194-206 (2018)

Mendoza, H. H., Burbano, V. M., y Valdivieso, M. A., El rol del docente de matemáticas en educación virtual universitaria. Un estudio en la Universidad Pedagógica y Tecnológica de Colombia, https://doi.org/10.4067/S071850062019000500051, Formación universitaria, 12(5), 51-60 (2019) 
Nori, W.M., Kassim, C., Ahmad, S., y Mohd, N., The use of accounting software for effective teaching and learning introductory accounting: A pedagogical discusión, https://doi.org/10.3923/sscience.2016.3315.3320, The social sciences, 11(1), 3315-3320 (2016)

Núñez, E., y Monclúz, I.M., El impacto de la utilización de la modalidad b-Learning en la educación superior, https://doi.org/10.17163/alt.v14n1.2019.02, Alteridad Revista de Educación [Internet], 4(1), 26-39 (2019)

Rosales, F. G., Mercado, V. M., Monasterolo, R.R., y Ribotta, S.L, Implementación de un laboratorio de física en tiempo real para el aprendizaje activo de circuitos eléctricos, https://doi.org/10.4067/S0718-50062012000300002, Formación Universitaria, 9(6), 3-12 (2016)

Salazar, O. M, Ovalle, D. A., y Duque, N. D., Evaluación del desempeño basado en métricas de un sistema pedagógico multi-agente, ubicuo sensible al contexto y apoyado en ontologías, https://doi.org/10.4067/S0718-50062016000300003, Formación universitaria, 9(3), 11-22 (2016)

Sánchez, M., Garcia, J., Steffens, E., y Hernandez, H., Estrategias pedagógicas en procesos de enseñanza y aprendizaje en la educación superior incluyendo tecnologías de la información y las comunicaciones, https://doi.org/10.4067/S071807642019000300277, Información tecnológica, 30(3), 277-286 (2019)

Seixas, S., Dove, C., Ueberschär B., y Bostock, J., Evaluation on the use of e-learning tools to support teaching and learning in aquaculture and aquatic sciences education, https://doi.org/10.1007/s10499-014-9828-9, Aquaculture International, 23(3), 825-841 (2015)

Slobodan, M., y Todorović, M., How does management accounting change under the influence of ERP?, https://doi.org/ 10.1080/1331677X.2012.11517531, Economic Research-Ekonomska Istraživanja, 25(3), 722-751 (2012)

Soler, J., Prados, F., Poch, J., y Boada, I., ACME: Plataforma de aprendizaje electrónico (e-learning) con funcionalidades deseables en el ámbito de la ingeniería, https://doi.org/10.4067/S0718-50062012000300002, Formación universitaria, 5(3), 3-16 (2012)

Trujillo, F.S., y Salvadores, C.M., Tecnología para la enseñanza y el aprendizaje de lenguas extranjeras: revisión de la literatura, https://doi.org/10.5944/ried.22.1.22257, RIED Revista Iberoamericana de Educación a Distancia, 22(1), 153-169 (2019)

Wright, S., Forray, J.M., y Lund, K., From advocacy to accountability in experiential learning practices, https://doi.org/10.1177/1350507618814645, Management Learning, 50(3), 261-281 (2019) 